\title{
Application of gradient adaptive lattice filters to channel equalisation
}

\author{
P.M. Grant and M.J. Rutter
}

Indexing term: Filters and filtering

\begin{abstract}
In this paper, the potential application of adaptive lattice equalisers based on gradient search adaptive algorithms, is examined. The assessment is based on simulations of equaliser performance in data communications systems which provides a comparison of the relative performance of these adaptive lattice equalisers with transversal equalisers. It highlights the critical balance between rapid convergence and degradation due to algorithm noise, which is involved when selecting the algorithm stepsize. Two new adaptive equaliser approaches are suggested, one based on a timed lattice structure feeding a linear combiner. The other is a short timed lattice structure in cascade with a transversal equaliser. Both approaches are shown to offer fast converging adaptive equalisers for data communications applications, which have a converged error performance superior to that of the direct application of a gradient adaptive lattice equaliser.
\end{abstract}

1

Fig. 1 shows the generalised schematic diagram of an adaptive filter [1] to which a distorted signal $s(t)$ is input.

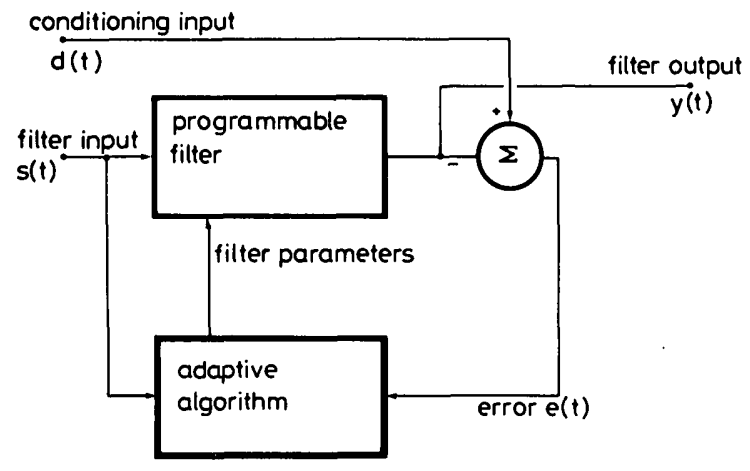

Fig. 1 Generalised block diagram of an adaptive filter

This is filtered to give an output $y(t)$, which is subtracted from a training signal $d(t)$ (sometimes called the desired or conditioning signal) to yield an error $e(t)$.

$$
e(t)=d(t)-y(t)
$$

This error is subsequently used in an adaptive algorithm [1] which updates the filter parameters to minimise the error. This structure is widely used in communication as an adaptive equaliser to minimise transmission distortion [2]. Other applications include the cancellation of echoes due to mismatches in the 2-4 wire hybrid transformer used in telephony [3] and cancellation of CW interference [4] or of noise [1], in high-interference environments such as aircraft cockpits and close to large motors.

The programmable filter most commonly used in adaptive processing is the finite-impulse-response (FIR) transversal structure [5], Fig. 2, and the most popular adaptive algorithm for controlling the filter weights is the leastmean-squares (LMS) stochastic gradient search approach first reported, in the context of adaptive filters, by Widrow [1]. Such adaptive filter designs are extensively reported in the literature and are widely applied in practical communications equipment, ranging from speech-band data modems [6] to wideband microwave digital radio systems [7]. In many of these applications the need for a separate

Paper 3054F (E7, E10), first received 11 th July 1983 and in revised form 11 th January 1984

The authors are with the Department of Electrical Engineering, University of Edinburgh, King's Buildings, Edinburgh EH9 3JL, Scotland training signal is overcome by differencing the filtered output with the detector output, after the decision process, in order to derive the error signal $[6,7]$.

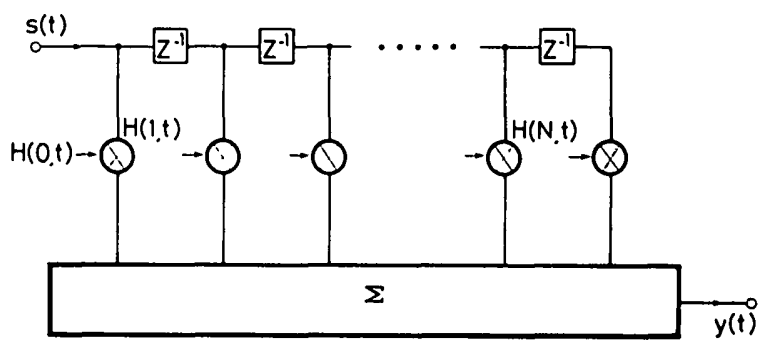

Fig. 2 Finite impulse response, transversal filter

These FIR adaptive filter structures are relatively easy to implement and are not too computationally demanding. Hardware and algorithm complexity rises linearly as a function of the order of the filter. However, their rates of convergence and ability to track non-stationary received signals are heavily dependent on the parameters of the communications channel itself.

Kalman estimation $[8,9]$ based structures avoid this problem, but at the expense of increased complexity. In these structures the computational load can be shown to increase as the cube of the filter order. Similar figures are quoted for matrix inversion based adaptive processors [10]. Fast Kalman techniques [11] are also attractive as they are not quite as computationally demanding, as accuracy of the arithmetic may be reduced. Their overall complexity may be close to lattice filter approaches reported here.

This paper is concerned with equaliser designs which are based on the gradient search adaptive lattice structure $[12,25]$. This structure is widely used in parametric spectral analysis [14] and linear predictive coding (LPC) of speech waveforms for bandwidth compression [15]. Gradient lattice equalisers offer a convergence rate and complexity which falls between the stochastic gradient algorithm transversal and Kalman techniques.

Designs of lattice processors based on exact leastsquares estimation techniques [14] have also been reported for use in equalisation $[16,17]$. These use techniques similar to Kalman estimation to calculate the filter coefficients. This requires division and hence is computationally much more demanding than the adaptive gradient search lattice approach reported in this paper. Our approach is better viewed as an extension of gradient search adaptive transversal filter techniques where the lattice filter is incor- 
porated to precondition the signal before it enters the weighted combiner. The primary aim of the paper is to use this slightly more sophisticated filter structure to provide faster initial convergence or, alternatively, superior tracking performance on ill conditioned channels.

A hardware lattice equaliser design has been previously reported as a TTL breadboard $[18,19]$ which offers a 16stage equaliser at $17 \mathrm{kHz}$ sample rate. It employed 12-bit input quantisation with 24-bit filter coefficient accuracy. A separate study on a bit-serial design for $5 \mu \mathrm{m}$ feature size NMOS process shows that a five-chip VLSI set is capable of processing signals at $20 \mathrm{kHz}$ sample rate for a 16-stage lattice filter $[19,20]$. Other integrated structures have reported alternative designs at slightly slower sample rates [21].

\section{Adaptive lattice equaliser designs}

The transversal filter of Fig. 2 comprises a linear combiner structure fed from a shift register. In the simplest lattice equaliser realisation (Fig. 3) the shift register is replaced by

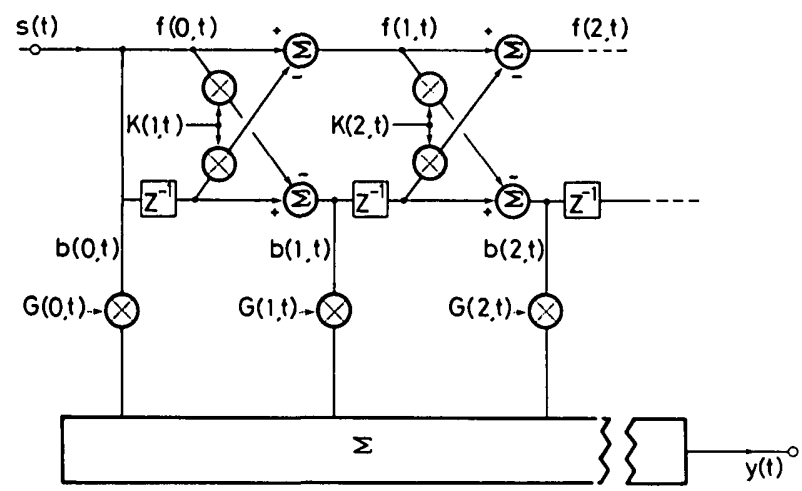

Fig. 3 Adaptive lattice equaliser employing a global combining structure

the lattice filter structure, which is also adaptive. The latter processes the input signal, using a set of PARCOR coefficients $(K(1, \ldots, N))$, whose values are obtained by an iterative adaptive recursion procedure. The equations governing the operation of the adaptive lattice structure are given in Table 1 . The $b$ outputs of the different stages are those of a family of prediction-error filters of increasing order. Thus all the respective $b(n, t)$ outputs can be shown to be orthogonal to each other as well as the input signal [13].

The process of orthogonalisation is also a spectral whitening process so the output from the final stage of the lattice structure is a noise-like signal. The PARCOR coefficient values thus contain certain information regarding the spectral properties of the input signal. In the linear predictive coder used for speech bandwidth compression [15], the whitening filter (PARCOR) coefficients values are transmitted to provide a time varying model of the speech signal. This is an example of one use of the adaptive lattice filter as a parametric spectrum analyser.

The problem inherent in the adaptive transversal filter is that the intersymbol interference (ISI) caused by the distorting channel in turn causes interaction between the convergence of the individual tapweights. This slows down the convergence of the adaption process in the combiner. The modes of convergence of a combiner structure have been related [22] to the eigenvalues of the cross-covariance matrix of the input signals, which in this case is the channel autocovariance matrix whose eigenvalues are closely related to the input power spectrum [23]. The optimum stepsize is inversely proportional to the mean
Initialisation:

$$
\begin{aligned}
& K(1, \ldots, N, 0)=0 \\
& b(1, \ldots, N, 0)=0 \\
& \mu(1, \ldots, N, 0)=0.002 / E\left\langle S^{2}(t)\right\rangle \quad \text { suggested values }
\end{aligned}
$$

Lattice signals:

$$
\begin{aligned}
& f(0, t)=b(0, t)=s(t) \\
& f(n, t)=f(n-1, t)-K(n, t) \cdot b(n-1, t-1) \\
& b(n, t)=b(n-1, t-1)-K^{*}(n, t) \cdot f(n-1, t)
\end{aligned}
$$

$K^{*}(n, t)$ in the complex conjugate of $K(n, t)$ but for processing of real signals $K(n, t)=K^{*}(n, t)$

Stepsize recursion:

$$
\begin{aligned}
1 / \mu(n, t)= & (1-a) \cdot(1 / \mu(n, t-1) \\
& +\beta \cdot\left(|f(n-1, t)|^{2}+|b(n-1, t-1)|^{2}\right.
\end{aligned}
$$

where constants $\alpha=0.02$ and $\beta=1$

PARCOR recursion:

$$
\begin{aligned}
& K(n, t+1)=K(n, t)+\mu(n, t) \\
& \left(f(n, t) \cdot b^{*}(n-1, t-1)+b^{*}(n, t) \cdot f(n-1, t)\right)
\end{aligned}
$$

power of the input [24] and the rate of convergence of a mode is proportional to the product of its eigenvalue and the single stepsize. Thus a small eigenvalue shows the rate of convergence of that mode, restricting the overall filter convergence.

The lattice structure is deployed to alleviate these problems. It preprocesses the signal and supplies a set of orthogonal signals to the combiner structure, whose amplitudes are related to the channel eigenvalues. The updating of the weights on each tap of the combiner in the lattice equaliser is then given a stepsize which is inversely proportional to signal power at the corresponding lattice output to give all taps the same rate of convergence [25]. The equaliser's overall rate of convergence is thus made independent of input channel characteristics. This is mathematically equivalent to normalising the signal power at the output of each stage. An alternative viewpoint is that in providing orthogonal signals, the lattice has removed the tapweight interaction and allowed a more reliable rate of convergence. It has recently been shown [26] that the time domain lattice structure is equivalent to the space domain Gram-Schmidt preprocessor [27] used in adaptive arrays, hence it is appropriate to study lattice structures further for fast converging adaptive equalisers.

There are several variants of the detailed design of gradient search adaptive lattice equalisers. One design combines all the lattice outputs in the single global combining structure of Fig. 3, which is similar to that used in the transversal filter (Fig. 2). This results in the same error signal being used in each tapweight update loop. For the same number of delay stages both the structures of Figs. 2 and 3 will attempt to converge to the same FIR filter solution with identical impulse response values. The transversal filter impulse response is controlled directly by the combiner weight values $H(N, t)$ while the response of the lattice equaliser, Fig. 3, is dependent on both $K(N, t)$ and $G(N, t)$ values. These can be shown [19] to be directly related to the $H(N, t)$ values.

An alternative structure [19] is the distributed combiner 
of Fig. 4 where individual error signals are derived for each of the update loops. This structure is more suitable for
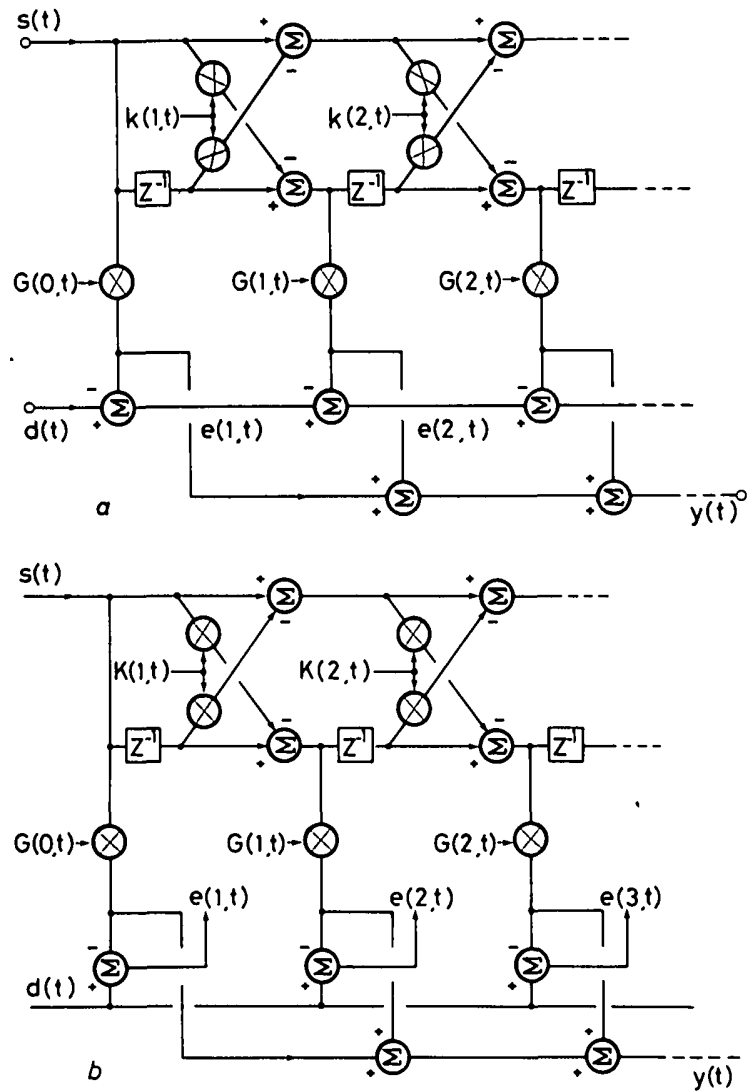

Fig. 4 Adaptive lattice equaliser employing a distributed combining structure

a Order-dependent combiner

$b$ Order-independent combiner

hardware multiplexing than the global type, but suffers from an increased level of algorithm noise. There are further variants [19] on the distributed combiner, which may be chained into order dependent structure (Fig. $4 a$ ) or separated into an order independent structure (Fig. $4 b$ ). The equations governing the operation of these adaptive combiner structures are given in Table 2. Note that these combiner structures also use the lattice stepsize recursion of Table 1 to set the value of $\mu(n, t)$.

\subsection{Rate of convergence}

The factors which control the rate of convergence of an adaptive transversal filter are the magnitude of the input

Table 2: Linear combiner equations

Initialisation:

$$
G(0, \ldots, N, t)=0
$$

Combiner signals:

$$
\begin{aligned}
y(0, t) & =0 \\
y(n+1, t) & =y(n, t)+G(n, t) \cdot b(n, t)
\end{aligned}
$$

Order-dependent distributed combiner error signal:

$$
\begin{aligned}
e(0, t) & =d(t) \\
e(n+1, t) & =e(n, t)-G(n, t) \cdot b(n, t)
\end{aligned}
$$

Global combiner error signal (alternative to above)

$$
e(n, t)=d(t)-y(N, t)
$$

Combiner weight recursion:

$$
G(n, t+1)=G(n, t)+2 \cdot \mu(n, t) \cdot b^{*}(n, t) \cdot e(n+1, t)
$$

signal and the stepsize, used in the tapweight update calculation. When the stepsize selected is dependent on the mean power input to the filter [24], i.e. the mean of the eigenvalues [23], the rate of convergence is largely determined by the value of the minimum eigenvalue. This is further illustrated in Fig. 5, which shows convergence plots

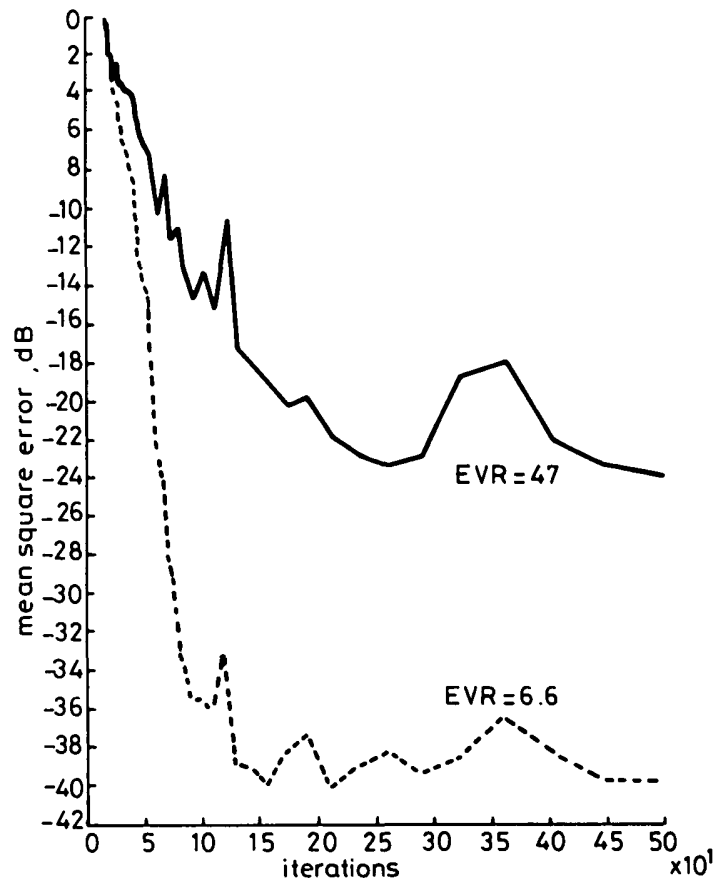

Fig. 5 Convergence plots for an 8-tap real LMS adaptive transversal equaliser when the distorting channel response has an eigenvalue ratio (EVR) of 6.6 (dotted curve) and 47 (solid curve)

for a real 8-tap adaptive transversal equaliser employing a fixed stepsize. The distorted signal used in the dotted curve has an eigenvalue ratio (EVR) or ratio of maximum to minimum eigenvalue of 6.6. In the upper solid curve the higher eigenvalue ratio of 47 gives a slower convergence. This is a similar property to jammer cancellation in adaptive antennas where high-level signals provide a higher loop gain and converge to a null more rapidly than the lowlevel signals [27]. These simulations show typical convergence characteristics by plotting instantaneous error power under noise-free conditions. Local deviations are introduced by the standard pseudo noise data which was transmitted. These can be removed by ensemble averaging, but this is not used in our simulation in order to economise on computer run time.

The convergence behaviour of the lattice equaliser is more complicated as one must consider the convergence of both PARCOR $(K)$ and combiner $(G)$ coefficients, Figs. 3 and 4 and Tables 1 and 2. The latter cannot start approaching the correct values until the former have converged towards their final solution, and the higher-order PARCOR coefficients are also affected by the convergence of lower-order values. Luckily, it has been shown that the PARCOR coefficients usually reduce in value with increasing order [19], minimising the delaying effect of the settling of the early stages on the values of later stages.

It is necessary to select appropriate stepsizes for both PARCOR and combiner coefficient calculations. Investigations have previously been conducted into the optimum stepsizes for the PARCOR recursion [28] and the results suggested that each stage should be designed for the same rate of convergence, i.e. with a stepsize inversely proportional to its power input. This has now been extended 
[19] to obtain the optimum ratio of PARCOR to combiner stepsizes, again assuming equal rate of convergence within the PARCOR and combiner coefficients. Fig. 6

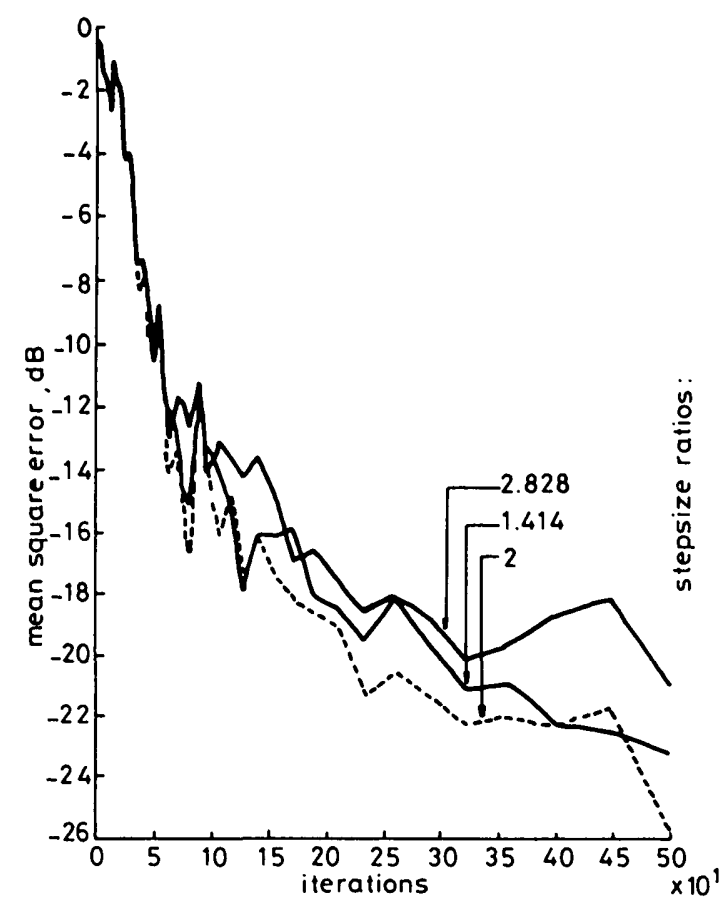

Fig. 6 Convergence properties of a 16-tap complex gradient adaptive lattice equaliser converging on a complex channel of eigenvalue ratios equal to 20

Three ratios of stepsize in the combiner to lattice structures are shown. A stepsize ratio of 2 is given in dotted curve while ratios of 1.414 and 2.828 are shown as solid curves

shows a typical set of equaliser convergence results for different ratios of the PARCOR to combiner stepsizes. The conclusion drawn, from these and other simulations [19], was that the combiner coefficient calculation had to have twice the stepsize of the PARCOR coefficients; hence the multiplication by two in the combiner recursion in Table 2. This difference arises from the fact that the PARCOR recursion has two correlation terms, while the combiner recursion employs only one. Hence a factor of two ensures equal convergence rates for both coefficients.

The rate of convergence is only part of the problem as it has also been found that the magnitude of the final converged error is also dependent upon the stepsize. After a gradient search adaptive filter has converged, its coefficients will deviate from those of the optimum Wiener filter by a small amount. This small random deviation arises from the stochastic nature of the self-adjustment process and the resulting increase in error is called algorithm noise. It has been shown [24] that the additional error due to algorithm noise is proportional to both stepsize and the ideal error output of the Wiener filter as given in [24]

$$
E\left\langle e^{2}\right\rangle=2 e^{2}(o p t) / 2(2-\mu N R(0))
$$

Where $e^{2}(o p t)$ is the mean-square error of the Wiener filter, $E\left\langle e^{2}\right\rangle$ is the actual filter mean-squared output, $\mu$ is the stepsize, $N$ is the number of taps and $R(0)$ is the meansquared value of the signal input.

Ungerboeck [24] has also derived a formula for calculating the stepsize for a tap in global combiner structure, to give near-optimum rate of convergence

$$
\mu=\frac{1}{N P}
$$

where $P$ is the mean power input to the tap. This rapid convergence is offset by an algorithm noise which equals, and thus doubles, the output error power. Global combiners used in equaliser applications typically have converged errors below $-20 \mathrm{~dB}$, and an additional $3 \mathrm{~dB}$ will not degrade operation unduly. Nevertheless, if it is necessary to approach the optimum even more closely, the combiner stepsize may be reduced at a later stage in the convergence.

Unfortunately, a gradient lattice stage may be also regarded as a one-tap adaptive filter, where the output of the stage is the adaptive filter error signal. Selecting a stepsize using eqn. 3 adds to the output signal an equal amount of stochastic algorithm noise. Ungerboeck's [24] equation for algorithm noise converts into lattice symbols as:

$$
Q(n)=\frac{\left(1-|K(n)|^{2}\right) Q(n-1)}{1-0.707 \mu(n, t) Q(n-1)}
$$

where $Q(n)$ is the mean-square value of $b(n, t)$ i.e. the power out of the lattice stage.

The resulting degradation in signal/noise ratio as one progresses down the lattice structure is seldom acceptable and a lower stepsize must be used. The noise introduced into the lattice structure decreases as the value of the PARCOR coefficient, $K(n)$, approaches its bounds of \pm 1 [21]. This noise on the lattice structure outputs increases on progression to higher orders, which introduces further errors in the target values towards which the later PARCOR coefficients are converging. Further noise is subsequently added by the adaptive combiner structure.

Fig. 7 shows a comparative plot of mean-squared error

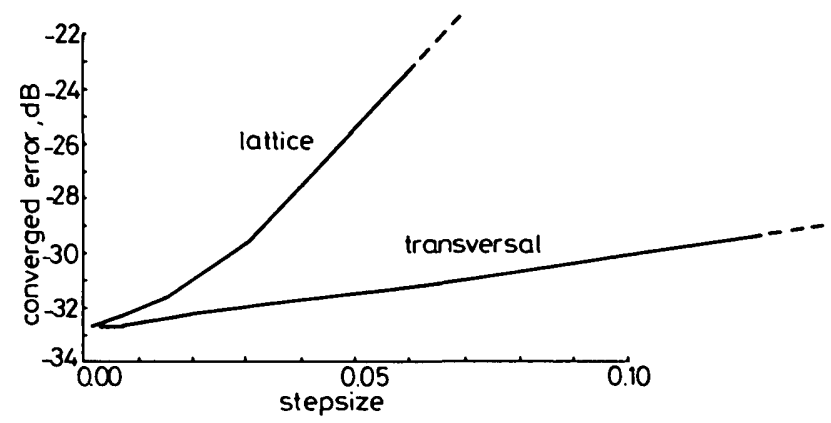

Fig. 7 Converged error against stepsize for 12-tap fixed stepsize adaptive transversal and lattice equalisers

output from lattice and transversal filters for various stepsize. The lattice algorithm deployed in these measurements used the order-dependent distributed combiner structure, Fig. $4 a$, with a fixed stepsize and not one which is inversely proportional to signal power. Nevertheless, it is clear that for a stepwise of $>0.01$ the algorithm noise of the lattice equaliser is much larger than in the transversal equaliser. As the stepsize is reduced to $<0.01$ both curves tend asymptotically towards the same Wiener solution, for this 12-tap equaliser.

For optimum rate of convergence the transversal filter stepsize for this case is given by eqn. 3 as $\mu=0.1$. Fig. 7 shows that this value of $\mu$ cannot be used in the lattice without incurring an excessive noise penalty. Thus the lattice stepsize must be reduced to a value close to $\mu=0.01$ to obtain a converged error performance equivalent to the transversal filter. Thus stepsize values close to Ungerboeck's optimum [24] cannot be used in practice in the lattice to obtain fast convergence without incurring a significant noise penalty.

These deficiencies are further illustrated in Fig. 8, which shows convergence plots for fixed stepsize complex lattice 
(dotted) and transversal (solid) equalisers with orders (numbers of delay stages or combiner taps) between 8 and

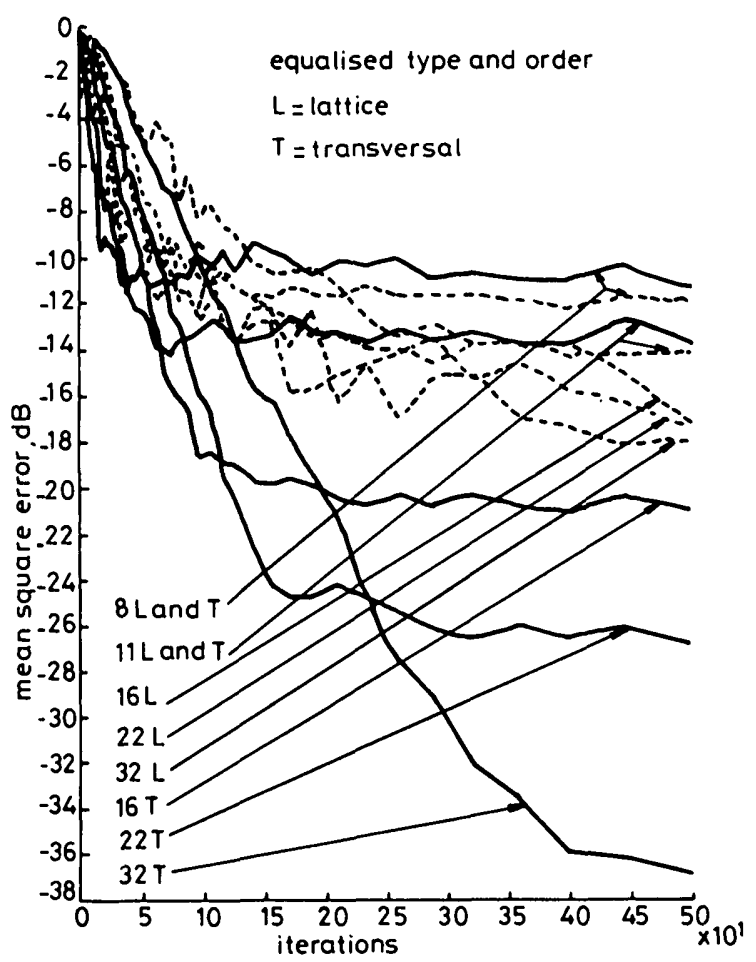

Fig. 8 Convergence properties for fixed stepsize complex adaptive lattice (dotted) and transversal (solid) equalisers of 8, 11, 16, 22 and 32 tap complexity when operating on a complex channel of low distortion (eigenvalue ratio equal to 8 )

32 , on a channel with an eigenvalue ratio of 8 . This complex channel is typical of a severely distorted UK telephone channel. The lattice equalisers generally show faster initial convergence but in the transversal equaliser the final converged error is seen to improve considerably when higher-order filters are used. In the lattice equaliser, however, the algorithm noise in the lattice structure provides a 17-18 $\mathrm{dB}$ floor which removes the benefit of the higher-order filters. The same floor persists when the length of the structure is increased beyond 16 stages, as the increase in the number of stages contributing to the noise is offset by the stepsize reduction according to eqn. 3 , maintaining the same $17-18 \mathrm{~dB}$ noise level. The lattice equaliser stepsize could be further reduced to improve the algorithm noise, but the convergence would then be slower than the transversal filter, making it a more complex and cumbersome structure with no particular advantages.

It is a feature of the adaptive stepsize algorithm, Table 1 , that the stepsize, in addition to being adjusted relative to the normalised signal power at each stage, may also be given an artificially high initial value. The entire stepsize vector will then tend asymptotically to its steady-state value as the convergence progresses. The result is a rapid initial convergence towards the optimum coefficient values, followed by a slower approach to a tapweight vector very close to the Weiner solution. Fig. 9 compares such an approach against the convergence of an 8-tap transversal filter with a fixed optimum stepsize of 0.125 . The perfect Weiner solution for the transversal equaliser on this channel was calculated as $-24.4 \mathrm{~dB}$ and the simulated dotted curve is shown to approach within $3 \mathrm{~dB}$ of this figure as suggested in Reference 24. The 8-tap lattice structure of Fig. $4 a$ initially employed a fixed stepsize of two times the optimum value, eqn. 3 , in each stage. This value was then reduced during the stepsize recursion to 0.25 of the transversal optimum value, over the first 50 iterations. This achieves a faster convergence for the solid lattice filter

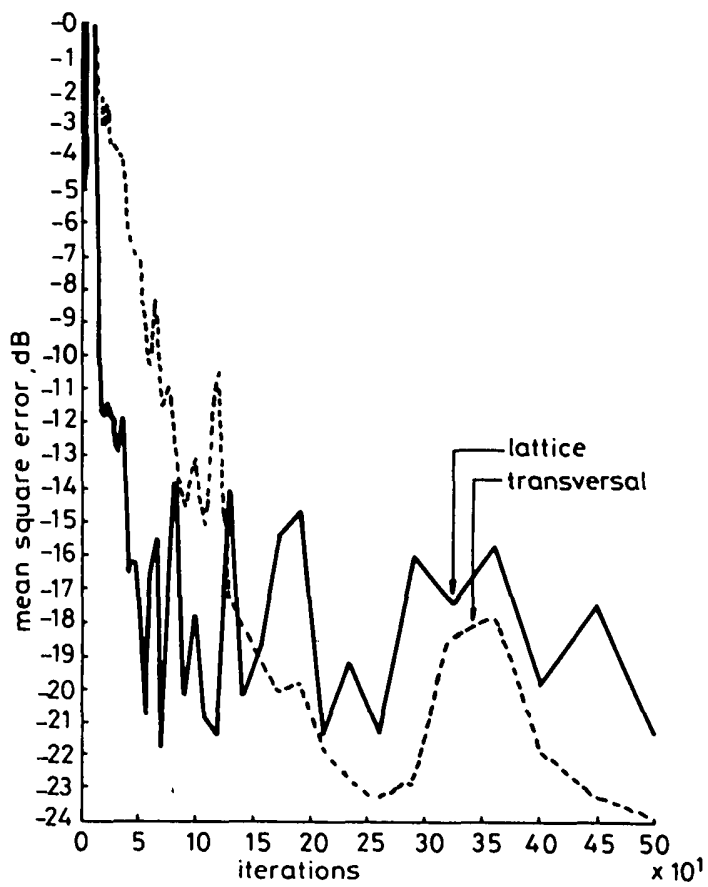

Fig. 9 Convergence properties of transversal (dotted) and lattice (solid) equalisers for a real channel with high distortion (eigenvalue ratio of 47)

Transversal filter uses a fixed stepsize of 0.125 . Lattice commences with initial value of 0.25 on each stage, for fast initial convergence and subsequently reduces to 0.25 the optimum transversal value over the first 50 iterations to minimise algorithm noise

characteristic, but it still introduces excess noise in the converged state. The distorting channel deployed in these simulations was real rather than complex. It was the synthetic channel used earlier in Fig. 5 which had an impulse response of $0.4+z^{-1}+0.4 z^{-2}$, giving an eigenvalue ratio for 8-tap equalisers equal to 45 .

It was concluded from Fig. 9 and other similar simulations which were conducted [19] that, although the gradient lattice equalisers can provide faster initial convergence, their increased algorithm noise introduces degradation in the final convergence performance compared to simpler transversal filter based equaliser. Thus the higher complexity. of the lattice approach cannot be fully justified on these performance considerations.

\section{Alternative equaliser design}

One alternative design approach retained the distributed combiner structure of the normal lattice equaliser, but froze the lattice PARCOR coefficients after 10-30 iterations. This ensures that the lattice structure will be close to convergence and will have removed most of the correlation from the signals feeding the combiner weights. Freezing the PARCOR coefficients removes the algorithm noise contributed by the lattice structure. This was found to be only partially successful, when using the distributed combiner, Fig. 4, as the latter must be fed with truly orthogonal signals in order to operate effectively. Since the PARCOR coefficients were frozen prematurely this condition was not achieved. However, Fig. 10 shows results for such a timed algorithm using a global combiner, Fig. 3, in an 8-tap lattice equaliser. The same synthetic channel was used as in the simulation of Fig. 9. Fig. 10 shows that the lattice equaliser has a similar rate of initial convergence to the untimed lattice in Fig. 9, but considerable improvement is obtained in the converged error by freezing the 
lattice recursion after 15 iterations and employing the global combiner in place of the distributed combiner.

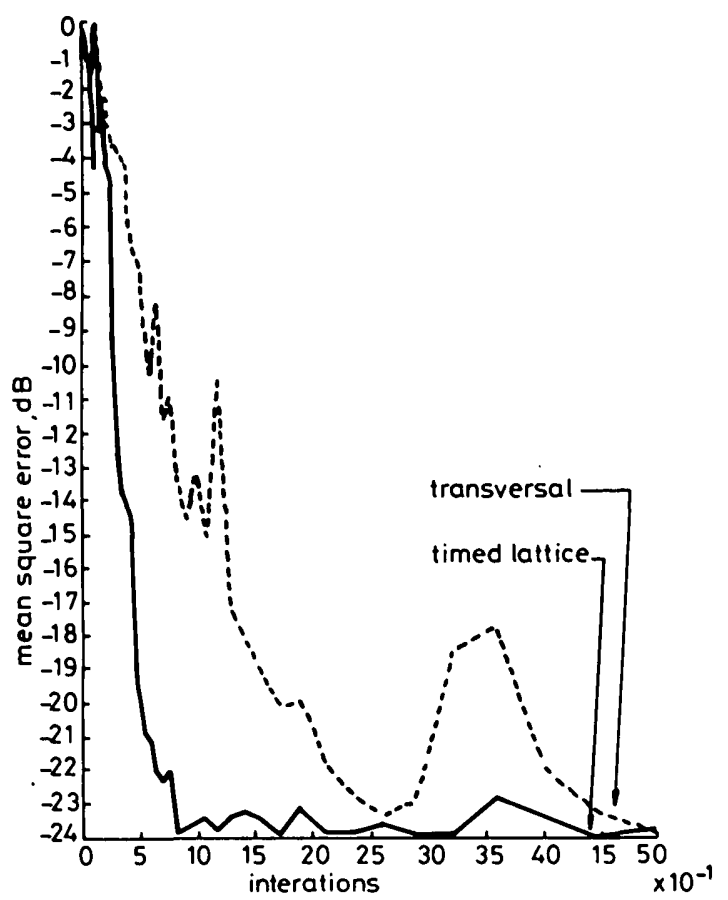

Fig. 10 Convergence properties of a timed lattice equaliser, employing the global combiner structure, Fig. 3

Here the lattice recursion to calculate the PARCOR coefficients is frozen after 15 iterations providing a significant reduction in the converged error compared to Fig. 9

A further concept was evaluated in which the highestorder output of an adaptive lattice filter structure preceded an adaptive transversal filter. Since it is a prediction-error filter, the lattice structure operates as a whitening filter and its final output reduces the overall eigenvalue ratio of the signal before it enters the transversal filter. The whitening of the input signal causes the transversal filter to converge faster as shown earlier in Fig. 5. Unfortunately, the prewhitening filter effectively spreads the channel impulse response in time, increasing the transversal filter order which is required to achieve a specific converged error. The maximum value on this time spread is equal to the order of the lattice filter. As it results from the convolution of the channel and converged lattice filter impulse responses the actual time spread is dependent on the converged lattice values.

It was found best to employ only a single-stage lattice filter, Fig. 11, to minimise the time spread and again freeze the PARCOR coefficients after 15-30 iterations to minimise algorithm noise. Fig. 12 shows a convergence curve for such an 8-tap combined equaliser, compared to a conventional transversal equaliser, for the same synthetic

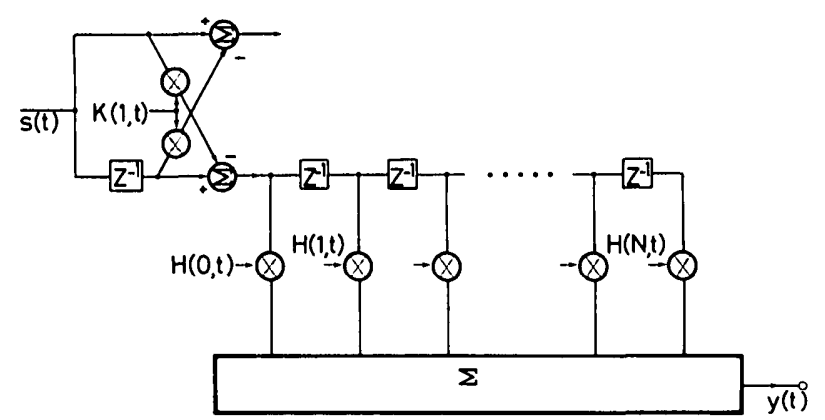

Fig. 11 Equaliser structure with single stage adaptive lattice prewhitener followed by conventional adaptive transversal filter channel as was used in Fig. 9. The lattice recursion was again frozen after 15 iterations. This provides an efficient

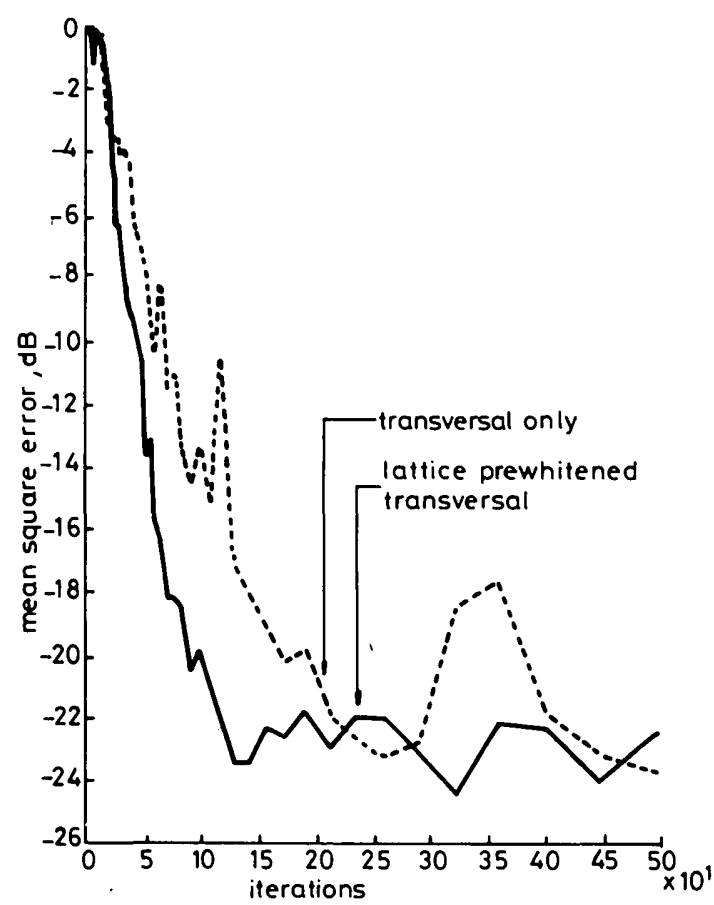

Fig. 12 Solid curve shows the results obtained by applying a single stage adaptive lattice prewhitening filter in advance of the 8 tap transversal filter. Lattice filter coefficients are again frozen after 15 iterations. Dotted curve shows equivalent transversal filter performance as shown earlier in Fig. 9

solution whose performance in terms of initial rate of convergence lies between the timed lattice equaliser and the basic transversal type. However as the prewhitened transversal equaliser structure had not been increased in length, to compensate for the time spread, there is a small degradation in the final converged error in Fig. 12 compared to the Fig. 10 results.

\section{Conclusions}

This paper has reported the design of adaptive equalisers based on gradient search transversal and lattice filter structures. The lattice structure has been suggested [25] to potentially offer significant improvements in terms of convergence and tracking rates through its ability to provide orthogonal signal samples which overcome the intertap correlation problems of adaptive transversal filters.

Although it is undisputed that the lattice filter is attractive for parametric spectral analysis [14] detailed theoretical assessment and simulation of its behaviour as an adaptive equaliser has shown significant problems with algorithm noise resulting in excessively large converged errors.

The final Section of this paper has examined other techniques to overcome these problems such as the timed approach where the lattice filter coefficients are frozen after initial convergence or the deployment of a small timed lattice prewhitener in advance of a conventional transversal filter. Both of these approaches have been shown to provide a significant reduction in the algorithm noise compared to nontimed lattices. The timed lattice approach also preserves the fast initial convergence of the adaptive gradient lattice structure. The simpler lattice prewhitener offers a initial convergence performance which is superior to the transversal filter but is marginally inferior to the timed lattice structure. Both approaches offer an 
overall complexity reduction compared to non-timed gradient lattices and are thus now worthy of further investigation in the context of specific applications.

\section{Acknowledgment}

The authors wish to acknowledge helpful discussion with C.F.N. Cowan and A. Morgul while conducting these studies, and also thank the anonymous referees for their comments and observations on the initial version of this manuscript. The sponsorship of the UK Science and Engineering Research Council is also gratefully acknowledged.

\section{References}

1 WIDROW, B., et al: 'Adaptive noise cancelling: principles and applications', Proc. IEEE, 1975, 63, pp. 1692-1716

2 CLARK, A.P.: 'Advanced data transmission systems' (Pentech Press, 1977)

3 SONDHI, M.M., and BERKLEY, D.A.: 'Silencing echoes on the telephone network', Proc. IEEE, 1980, 68, pp. 948-963

4 GRANT, P.M., and KINO, G.S.: 'Adaptive filtering based on SAW Monolithic storage correlator', Electron. Lett., 1978, 14, (7), pp. 562-564

5 KALLMAN, H.E.: 'Transversal filters', Proc. IRE, 1940, 28, pp. 302-310

6 WESTALL, F.A.: 'Efficient digital signal processing realisations for PSK modem receivers'. IERE Conf. Proc., 1981, No. 49, pp. 143-152

7 DUDEK, M.T., and ROBINSON, J.M.: 'A new adaptive circuit for spectrally efficient digital microwave-radio-relay systems', Electron. \& Power, 1981, 27, (5), pp. 397-400

8 KALMAN, R.E.: 'A new approach to linear filtering and prediction problems', Trans. ASME. J. Basic Eng., 1960, pp. 35-45

9 LAWRENCE, R.E., and KAUFMAN, H.: 'The Kalman filter for the equalisation of a digital communications channel', IEEE Trans., COM-19, pp. 1138-1141

$10 \mathrm{KUNG}$, S.Y., and HU, Y.H.: 'A highly concurrent algorithm and pipelined architecture for solving toplitz systems', ibid., 1983, ASSP31, pp. 66-75
11 FALCONER, D.D., and LJUNG, L.: 'Application of fast Kalman estimation to adaptive equalisation', ibid., 1978, COM-26, pp. 14391446

12 GRIFFITHS, L.J.: 'An adaptive lattice structure for noise-cancelling applications', Proc. IEEE ICASSP Conf., 1980, pp. 87-90

13 MAKHOUL, J.: 'Stable and efficient iattice methods for linear prediction', IEEE Trans., 1977, ASSP-25, pp. 423-428

14 FRIENDLANDER, B.: 'Lattice methods for spectral estimation', Proc. IEEE, 1982, 70, pp. 990-1017

15 BLANKENSHIP, P.E.: 'A review of narrowband speech processing techniques', IEE Conf. Publ. No. 180, Sept 1979, pp. 108-117

16 SATORIUS, E.H., and PACK, J.: 'Application of least squares lattice algorithm to adaptive equalisation', IEEE Trans., 1981, COM-29, pp. 136-142

17 LING, E., and PROAKIS, J.G.: 'Adaptive lattice decision-feedback equalisers and their application on fading dispersive channels'. IEEE Int. Commun. Conf. (ICC) Proc. 83CH 1874, July 1983, pp. 854-858

18 RUTTER, M.J., GRANT, P.M., and COWAN, C.F.N.: 'Adaptive lattice filters for channel equalisation'. IEE Colloquium Digest $82 / 52$, 1982, pp. 9-1 to 9-4

19 RUTTER, M.J.: 'The theory design and application of gradient adaptive lattice filters', Ph.D. thesis, University of Edinburgh, 1983

20 RUTTER, M.J., GRANT, P.M., RENSHAW, D., and DENYER, P.B.: 'Design and realisation of adaptive lattice filters', Proc. IEEE ICASSP Conf., April 1983, pp. 21-24

21 AHMED, H.M., MORF., M., LEE, D.T., and ANG, P.H.: 'A VLSI speech analysis chip set based on square root normalised ladder forms', Proc. IEEE ICASSP Conf., 1981, pp. 648-653

22 WIDROW, B.: 'Adaptive filters' in KALMAN, R., and DECLARIS, N. (ed.): 'Aspects of network and system theory' (Rinehart and Winston 1971), pp. 563-587

23 WIDOM, H.: 'Toeplitz matrices', in HIRSCHMANN, I.I. Jr. (Ed.): 'Real and complex analysis' (Mathematical Association of America, Prentice-Hall, 1965)

24 UNGERBOECK, G.: 'Theory of speed of convergence in adaptive equalisers for digital communications', IBM J. Res. \& Dev., 1972, 16, pp. 546-555

25 SATORIUS, E.H., and ALEXANDER, S.T.: 'Channel equalisation using adaptive lattice algorithms', IEEE Trans., 1979, COM-27, pp. 899-905

26 McWHIRTER, J.G., and SHEPHERD, T.J.: 'Adaptive algorithms in the space and time domains', IEE Proc. F, Commun., Radar \& Signal Process., 1983, 130, (1), pp. 17-21

27 MONZINGO, R.A., and MILLER, T.W.: 'Introduction to adaptive antennas' (John Wiley, 1980) 